\title{
Correlating the chronology and local structure of energy materials
}

$\underline{\text { Daniel Olds }}^{1}$, Jue Liu ${ }^{1}$, Joshua Kim ${ }^{1}$, Peter Peterson ${ }^{1}$, Keith Lawler ${ }^{2}$, Paul Forster ${ }^{2}$, Arnold Packlar ${ }^{3}$, James Neilson ${ }^{3}$, Katharine Page ${ }^{1}$ ${ }^{1}$ Spallation Neutron Source, Oak Ridge National Laboratory, Oak Ridge, United States, ${ }^{2}$ Department of Chemistry, University of Nevada, Las Vegas, NV, United States, ${ }^{3}$ Department of Chemistry, Colorado State University, Fort Collins, CO, United States E-mail: oldsdp@ornl.gov

With its high flux and wide-Q range coverage, the NOMAD diffractometer at the Spallation Neutron Source allows for in situ neutron total scattering studies of materials undergoing chemical processes and reactions with temporal resolution of minutes or seconds. By combining this unparalleled capability with a recently commissioned high-precision gas flow cell sample environment, we have followed both the average and local structure of two energy materials in operando through critical chronological processes. We explicitly resolve the location of the nitrogen adsorption site in Zeolite-X at room temperature and under 1 ATM gas flow. Our stroboscopic event-based data processing and isotope-variation procedures are complemented by N2 adsorption isotherms and Monte Carlo simulations. We present our studies on the structure and chronology of two lithium-ion battery polymorphs (ordered and disordered) of spinel LixNi0.5Mn1.504 during thermally induced failure, highlighting structure-property relationships relevant to safety improvements and material fatigue. Both these examples demonstrate a common challenge of in operando studies, the difficulty of predicting a priori precisely when and where a feature of interest is going to emerge. Such uncertain conditions can lead to incomplete data collection, facilitated the need for return or repeat measurements. We present a recently developed model-free analysis toolset to help facilitate real-time appraisal of total scattering data at-the-beamline.
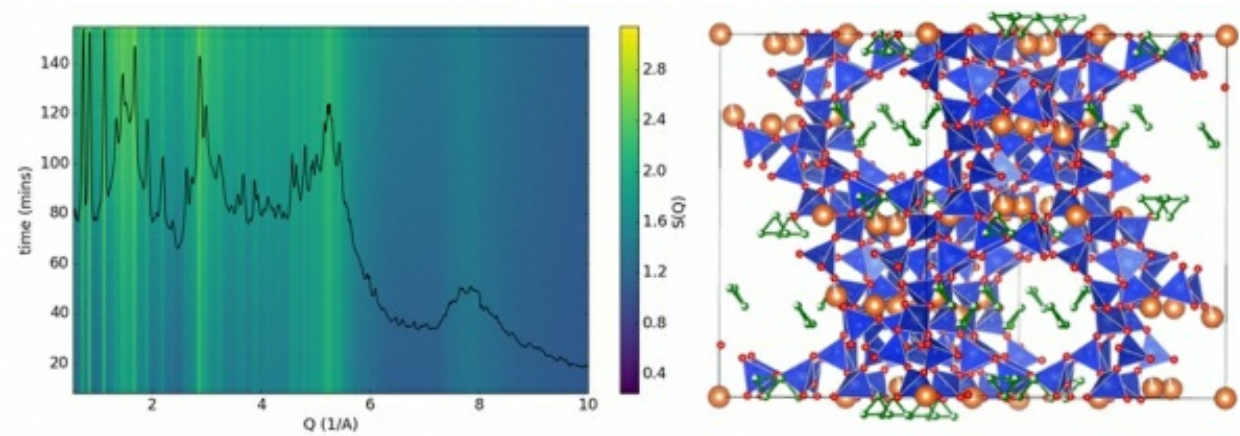

The measured neutron total scattering structure factor, $\mathrm{S}(\mathrm{Q})$, from zeolite-X material undergoing nitrogen adsorption (left). The average scattering curve is overlaid the instantaneous scattering as a function of total measurement time. By pulsing different isotopes of nitrogen, we were able to resolve the adsorption sites in the structure (right).

Keywords: Neutron Total Scattering, Gas flow catalysis, energy materials 